\section{Insulin injections and stimulation-bound feeding in the rat*}

\author{
H. R. BERTHOUD and K. BÄTTIG \\ Hygiene Institute, Behavioral Biology Laboratory \\ Swiss Federal Institute of Technology \\ Turnerstrasse 1, 8006 Zurich, Switzerland
}

An experiment was undertaken to investigate the influence of subcutaneously injected insulin upon lateral hypothalamic-induced feeding. Under high intensity (optimal) current conditions, insulin treatment reduced stimulation-bound food intake in almost all rats, in several of which this reduction was highly significant. These results support the findings of Hernandez \& Briese (1971), who observed a decrease of lateral hypothalamic self-stimulation after the injection of insulin.

Electrical stimulation of the lateral hypothalamus can elicit feeding (Bruegger, 1943), as well as many other behaviors (Valenstein, Cox, \& Kokolewski, 1969). Stimulated rats are highly motivated to perform instrumental responses which were previously rewarded by food (Morgane, 1961). Since "induced feeding" also depends on the palatibility of the food (Tenen \& Miller, 1964) and satiation (Devor, Wise, Milgram, \& Hoebel, 1970), and is decreased by amphetamine and other anorexigenic drugs (Stark \& Totty, 1967), one might believe that "induced hunger" is very similar to "natural hunger." Insulin injections, which produce hypoglycemia and, as a consequence, feeding (Booth \& Brookover, 1968; Steffens, 1969), might be expected to also increase electrically induced feeding. The glucostatic hypothesis seems to point in this direction (Mayer \& Thomas, 1967), particularly since insulin hypoglycemia has been shown to decrease the electrical activity of ventromedial, and sometimes to increase the activity of lateral hypothalamic, neurones (Anand, Chhina, Sharma, Dua, \& Singh, 1964).

Balagura \& Hoebel (1967) observed an increase of lateral hypothalamic self-stimulation with a very small dose (.025 units per rat) of insulin and a decrease with glucagon. Since a parallelism between the "rewarding" and the "hunger-inducing" effect of lateral hypothalamic stimulation had appeared in several studies (Margules \& Olds, 1962; Hoebel \& Teitelbaum, 1962), the authors suggested that insulin may act via its hypoglycemic effect and the hypothalamic "feeding areas" on positive reward.

Hernandez \& Briese (1971) observed a decrease of lateral

*This research was supported by Grant No. 3.33.68 from the Swiss National Foundation. The authors wish to thank Dr. P. Driscoll for his assistance in the preparation of the manuscript, and Helen Fehr and Armin Rhiner for their able technical assistance.
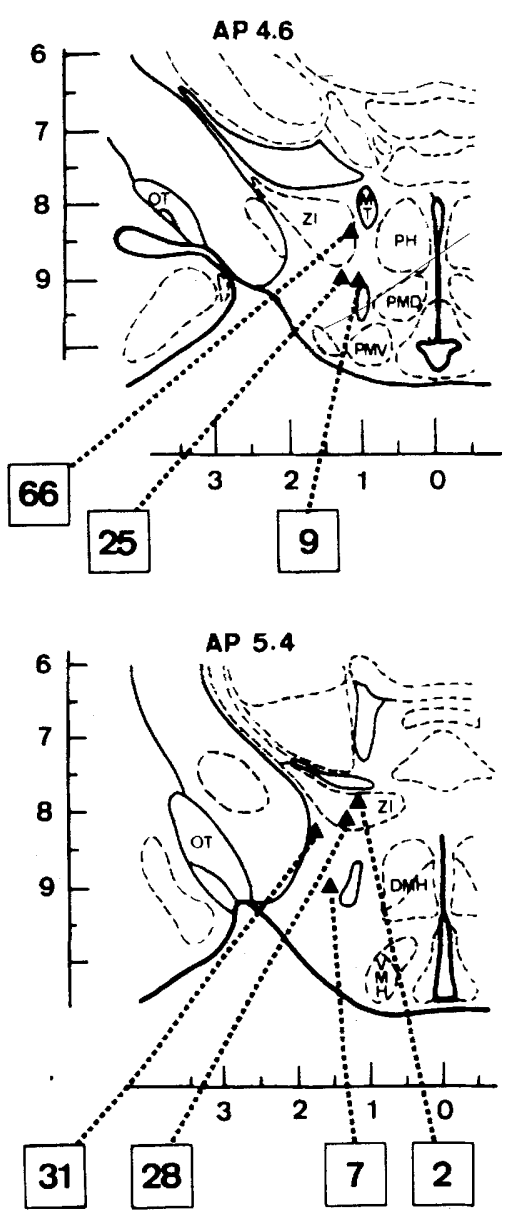
coordinates from De Groot. after pretreatment with insulin, Hernandez and Briese concluded that this pancreatic hormone regulates glucose entry into ventromedial glucoreceptors. Neural activity in the satiety center is thus increased, which exerts an inhibiting influence on the lateral hypothalamic feeding center.

This present study, therfore, was undertaken to investigate the influence of exogenous insulin upon lateral hypothalamic induced feeding, thus further defining the role that this hormone plays in hypothalamic functions.

\section{SUBJECTS}

Individually housed male albino rats, weighing between 280 and $300 \mathrm{~g}$ and about 3 months old, were bilaterally implanted with unipolar electrodes under pentobarbital anesthesia. With the bregma as a reference point, stainless steel electrodes of $0.3-\mathrm{mm}$ diam were lowered $7.7 \mathrm{~mm}$ from the dura and perpendicular to the skull, $1.9 \mathrm{~mm}$ posterior and $1.5 \mathrm{~mm}$ lateral. The ratio of the electrical conductive surface of
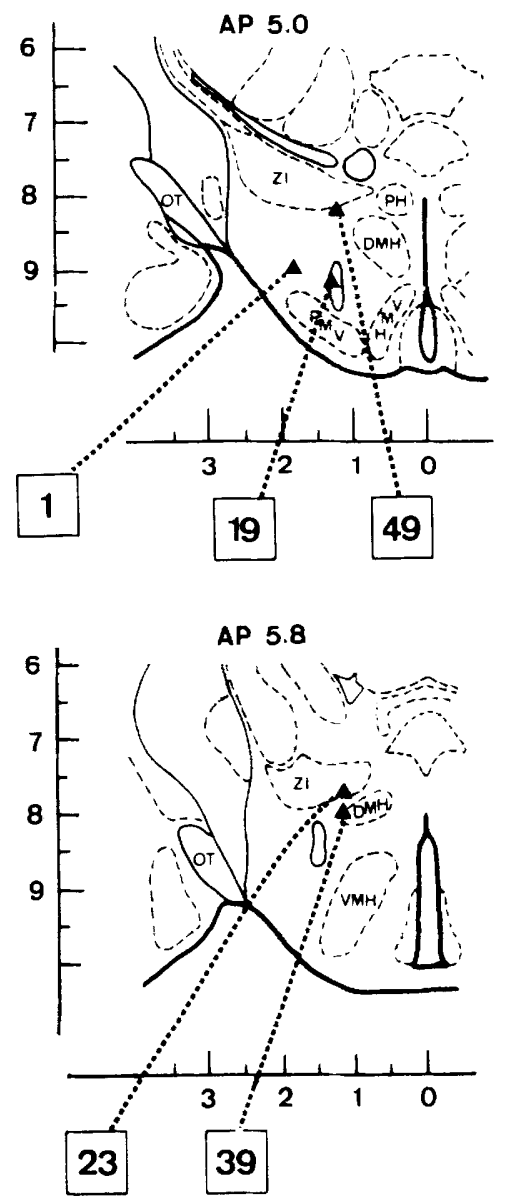

Fig. 1. Anatomical location of the electrode tips eliciting stimulation-bound eating. Points from the right hypothalamus are transferred to the left side. Drawings and abbreviations are from the Pellegrino and Cushman atlas, with AP 

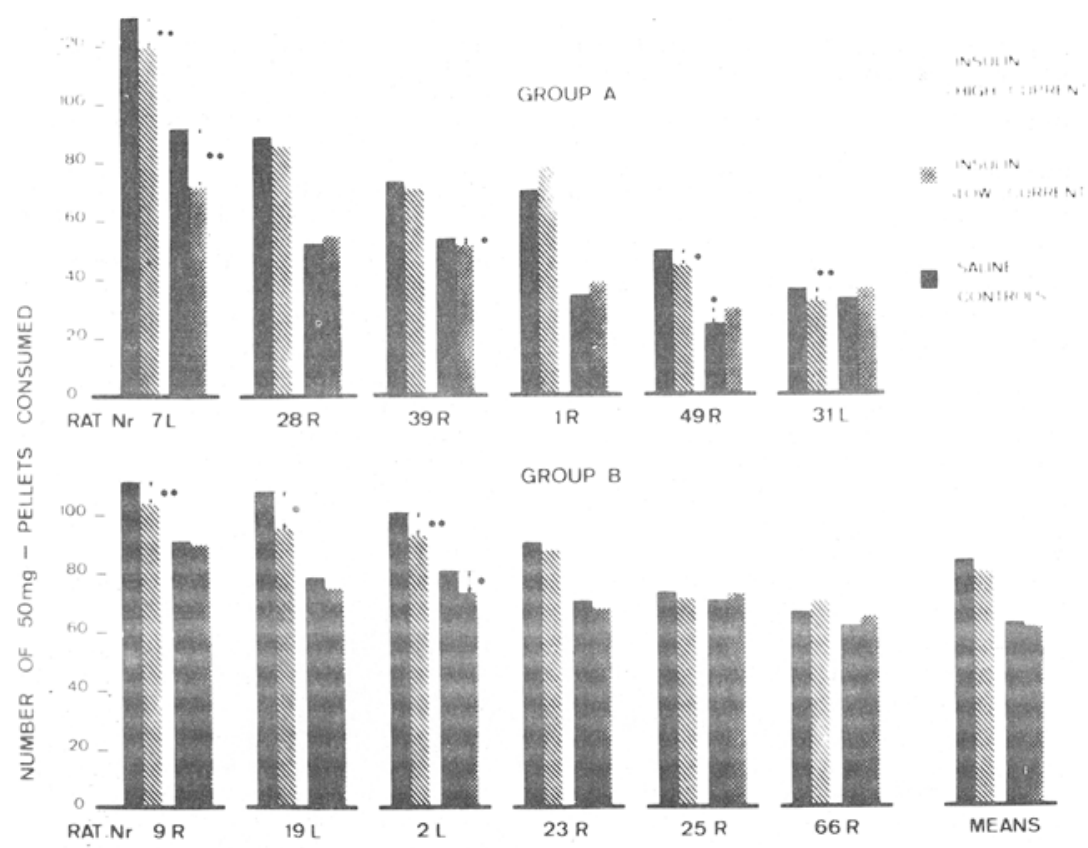

Fig. 2. Individual means of the number of food pellets consumed in one test session (10 x $60 \mathrm{sec}$ stimulation with 30 -sec intervals) under various conditions as indicated. Group A animals were run first with low-intensity and then with high-intensity stimulation. Group B animals were run in the reverse order. $(* \mathrm{p}<.05, * * \mathrm{p}<.005$.

each hypothalamic electrode to the indifferent electrode, consisting of two skull screws, a microconnector, and a linking copper wire, was about 1:10. APPARATUS

All testing was done in a cylindrical Plexiglas cage of $30-\mathrm{cm}$ diam. Monophasic rectangular pulses from a Philips pulse generator $(100 \mathrm{pps}, 2-\mathrm{msec}$ pulse duration) were controlled by a timer and delivered to the animal through a flexible cable and a swivel device, so that the rat could move freely. Peak-to-peak current intensity, monitored with a series resistance and an oscillograph, ranged from 50 to 250 microA. Fifty-milligram pellets (70\% starch, $30 \%$ lactose, flavored with vanillin) with a density of about 0.3 pellets $/ \mathrm{cm}^{2}$, scattered on the cage floor, served as test food.

\section{PROCEDURE}

In a preliminary testing period, the rats were checked for induced feeding from 8 to 16 days after surgery. Starting at 50 microA $A_{p p}$, current intensity was raised in 25-microA steps, until (1) the animals ate, (2) abnormal motor agitation was observed, or (3) a maximum current of 250 microA was reached. Stimulation train durations and interstimulus intervals both ranged from 30 to $60 \mathrm{sec}$, depending upon the strength of the induced behavior. About 15 sessions in 2- to 5-day intervals were performed, and at the end of screening, the current intensity thresholds for the stimulation-bound "eaters" were determined by a method of limits.

For the experiment proper, 12 rats that displayed reliable stimulation-bound eating were divided equally into two groups. Saline solution or 0.6 international units of neutral crystalline insulin from swine pancreas (Actrapid, Novo Industri, Copenhagen) were injected subcutaneously into the flank, $60 \mathrm{~min}$ before the start of the session. During this time all rats were deprived of food. This insulin dosage had been previously calculated to produce a fall in blood glucose concentration of at least $50 \mathrm{mg} / 100 \mathrm{ml}$ in $60 \mathrm{~min}$. In a first 8-day period, Group A animals were stimulated with a slightly suprathreshold current $(\mathrm{T}+10 \%$, range $5 \%-20 \%$ and Group $B$ animals with an optimal current $(\mathrm{T}+40 \%$, range $20 \%-60 \%$ ), this latter being defined as that which elicited maximal stimulation-bound eating without visible motor-stimulation effects. Each rat had four saline (control) and four insulin trials. In a following 8-day period, after a 4-day interval, current intensity conditions were reversed for the two groups. Daily tests consisted of 1060 -sec stimulation trains, each separated by $30-\mathrm{sec}$ intervals. The mean number of food pellets consumed under saline and insulin conditions were compared for each rat by a $t$ test.
At the completion of the experiment, the rats were perfused with isotonic saline and then with $10 \%$ Formalin, under deep pentobarbital anesthesia. The removed brains were frozen and sectioned into 25 micron cuts. Every fourth cut was mounted and stained with cresyl violet, and examined for the electrode traces. The anatomical locations of the electrode tips for all rats are shown in Fig. 1.

RESULTS AND DISCUSSION

Stimulation-bound food intake at high-intensity current was reduced in 10 out of 12 insulin-treated rats, as can be seen in Fig. 2. This decrease was highly significant in 5 animals $(p<.005)$ and moderately significant in one other $(p<.05)$. At stimulation with the slightly suprathreshold current, only 6 insulin-treated rats showed decreased intake, this decrease being in 1 case highly significant and in 2 cases moderately so. Since stimulation thresholds successively declined over the experiment as a whole, differences of intake due to low and high intensity of Group A rats were larger than those of Group B rats. Such a decline has been previously observed by Wise (1968). Depression of intake with insulin, and with both low- and high-intensity current, was most prominent in the 4 rats with the highest control intake (Rats $7 \mathrm{~L}, 9 \mathrm{R}$, $19 \mathrm{~L}$, and 2L). General depression of activity caused by insulin was never observed during this study, although our dose was more than 10 times that reported by Balagura \& Hoebel (1967).

In the present experiment, stimulation-bound eating was inhibited by insulin, this effect being more pronounced in situations with intense rather than weak stimulation-bound eating. This interaction between insulin effect and strength of elicited behavior cannot be explained on the basis of the present results. The inhibiting effect of insulin, however, is compatible with the hypothesis of Hernandez \& Briese (1971), in that insulin enhances glucose entry into the ventromedial hypothalamic nucleus (VMN), where glucoreceptors are activated that exert an inhibiting influence on the lateral hypothalamic area. In normal mice and rats, goldthioglucose injections lead to damage of the VMN and result in increased food intake and obesity (Marshall \& Mayer, 1954). In genetically or alloxan diabetic mice, however, VMN damage can be obtained by goldthioglucose application only after pretreatment with insulin (Debons, Krimsky, \& From, 1970). An and and his coworkers (1964) observed an initial increase of spike frequency in ventromedial hypothalamic neurons after IV injection of insulin in cats. 
Steffens (1969) found a latency of more than $60 \mathrm{~min}$ between SC injection of insulin and starting a meal in rats, in spite of the immediate hypoglycemia. All of these findings, together with the well-known hyperphagia of diabetics and the results of the present experiment, support the hypothesis of an insulin-dependent, glucosensitive mechanism in the VMN. There are, however, contradictory facts that remain unexplained, such as insulin-induced hyperphagia remaining in animals that became obese after VMN lesions (Epstein \& Teitelbaum, 1967; Steffens, 1969).

\section{REFERENCES}

ANAND, B. K., CHHINA, G. S., SHARMA, K. N., DUA, S., \& SINGH, B. Activity of single neurones in the hypothalamic feeding centers: Effect of glucose. American Journal of Physiology, 1964, 207, 1146-1154.

BALAGURA, S., \& HOEBEL, B. G. Self-stimulation of the lateral hypothalamus modified by insulin and glucagon. Physiology \& Behavior, 1967 , $2,337-340$.

BOOTH, D. A. \& BROOKOVER, T Hunger elicited in the rat by a single injection of bovine crystalline insulin. Physiology \& Behavior, 1968, 3, 439-446.

BR UEGGER, M . Fresstrieb als hy pothalamisches Symptom. Helvetia Physiologica Acta, 1943, 1, 183-198.

DEBONS, A. F., KRIMSKY, I., \& FROM, A. A direct action of insulin on the hypothalamic satiety center. American Journal of Physiology, 1970, 219, 938-943.

DEVOR, M. G., WISE, R. A., MILGRAM, N. W., \& HÖEBEL, B. G. Physiological control of hypothalamically elicited feeding and drinking. Journal of Comparative \& Physiological Psychology, 1970, 73, 226-232.

EPSTEIN, A. N., \& TEITELBAUM, P Specific loss of the hypoglycemic control of feeding in recovered lateral rats. American Journal of Physiology, 1967 , 213, 1159-1167.

HER NANDEZ, L., \& BRIESE, E. Insulin inhibition of hypothalamic self-stimulation. Acta Physiologica Latino Americana, 1971, 21, 57-63.

HOEBEL, B. G., \& TEITELBAUM, P. Hypothalamic control of feeding and self-stimulation. Science, 1962, 135, 375-376.

MARGULES, D. L., \& OLDS, J. Identical "feeding" and "rewarding" systems in the lateral hypothalamus of rats. Science, $1962,135,374-375$

MARSHALL, N. B., \& MAYER, J. Energy balance in goldthioglucose obesity. American Journal of Physiology, 1954. $178,271-274$

MAYER, J., \& THOMAS, D. W. Regulation of food intake and obesity. Science. $1967,156,328-337$.

MORGANE, P. J. Distinct "feeding" and "hunger motivating" systems in the lateral hypothalamus of the rat. Science, $1961,133,887-888$

STARK, P \& TOTTY, C. W Effects of amphetamines on eating elicited by hypothalamic stimulation. Journal of Pharmacology and Experimental Therapeutics, $1967,158,272-278$.

STEFFENS, A B The influence of insulin injections and infusions on eating and blood glucose level in the rat. Physiology \& Behavior, $1969,4,823-828$.

TENEN, S. S. \& MILLER, N. E. Strength of electrical stimulation of lateral hypothalamus, food deprivation, and tolerance for quinine in food. Journal of Comparative \& Physiological Psychology, $1964,58,55-62$.

VALENSTEIN, E. S., COX, V. C., \& KOKOLEWSKI, J. W. The hypothalamus and motivated behavior. In J. Tapp (Ed.) Reinforcement and behavior. New York: Academic Press, 1969. Pp, 242-287.

WISE, R A Hypothalamic motivational systems: Fixed or plastic neural circuits? Science, $1968,162,377-379$. 